\title{
Diseño de una aplicación móvil educativa a través de App Inventor para reforzar el proceso de aprendizaje en operaciones con números enteros
}

\section{Design of an educational mobile application through app inventor to reinforce the learning process in operations with whole numbers}

\author{
Cristhian Quishpe-López \\ Universidad Central del Ecuador, Quito, Ecuador \\ cpquishpe@uce.edu.ec \\ https://orcid.org/0000-0003-1578-2685 \\ Santiago Vinueza-Vinueza \\ Universidad Central del Ecuador, Quito,Ecuador \\ sfvinueza@uce.edu.ec \\ http://orcid.org/0000-0002-0818-6554
}

(Recibido: 02/03/2021; Aceptado: 15/03/2021; Versión final recibida: 15/04/2021)

Cita del artículo: Quishpe-López, C., y Vinueza-Vinueza, S. (2021). Diseño de una aplicación móvil educativa a través de app inventor para reforzar el proceso aprendizaje de operaciones con números enteros. Revista Cátedra. 4(2), 39-54.

\section{Resumen}

En la actualidad el desarrollo e implementación de nuevas tecnologías se ha vuelto necesario en todos los ámbitos, en la educación nace con la obligación de utilizar nuevas herramientas que ayuden a potenciar el aprendizaje. En esta propuesta se analiza el impacto de una aplicación móvil educativa diseñada en la plataforma App Inventor, que busca ayudar a reforzar el proceso de aprendizaje de operaciones de números enteros. Se aprovechó la facilidad de manejo que presenta la plataforma online gratuita, para estructurar de manera llamativa todo el contenido acorde al nivel académico del usuario.

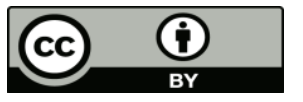


Así, se desarrollaron varias pantallas en la aplicación que lleva por nombre MatEstudio, cada una de estas pantallas sirven para fortalecer el proceso de aprendizaje. Estas pantallas se conforman por teoría, simuladores y un juego que en conjunto ayudan al razonamiento y retención del conocimiento. Se determinó el nivel de conocimiento teórico matemático y en Tecnologías de la Información y Comunicación de estudiantes de Educación General Básica. Posteriormente se aplicó una encuesta, en la cual, con base en los resultados obtenidos, se pudo evidenciar que se presenta una problemática para la correcta comprensión de la teoría y práctica de operaciones con números enteros. De esta manera se pudo concluir que es factible la implementación de una App Educativa creada en App Inventor para reforzar el proceso de aprendizaje de operaciones con números. No obstante, se presentan varias limitantes entre las principales el acceso a la red y la mala utilización del programa por parte del usuario.

\section{Palabras clave}

App Inventor, gamificación, aprendizaje, números enteros.

\section{Abstract}

At present the development and implementation of new technologies has become necessary in all areas, in education it is born with the obligation to use new tools that help to enhance learning. This proposal analyzes an educational mobile application designed on the App Inventor platform, which helps to reinforce the learning process of whole number operations. The ease of use of the free online platform was used to structure all the content in a striking way according to the academic level of the user. Thus, several screens were developed in the application called MatEstudio, each of these screens serves to strengthen the learning process. These screens are made up of theory, simulators, and a game that together help reasoning and knowledge retention. The level of theoretical mathematical knowledge and in Information and Communication Technologies of students of Basic General Education was determined. Subsequently, a survey was applied, in which, based on the results obtained, it was possible to show that there is a problem for the correct understanding of the theory and practice of operations with integers. In this way, it was concluded that it is feasible to implement an Educational App created in App Inventor to reinforce the learning process of operations with numbers.

\section{Keywords}

App Inventor, gamification, learning, whole numbers.

\section{Introducción}

La creación de aplicaciones móviles a través de App Inventor para el ámbito educativo ha crecido exponencialmente en los últimos años, dejando un poco de lado el uso de gran cantidad de libros u otros materiales. Con respecto a la plataforma, Almaraz et al. (2016) mencionan "la tecnología App Inventor se ha revelado como una herramienta accesible y potente para familiarizarse con la creación de aplicaciones para móviles. Su potencial para la creación de aplicaciones de contenido didáctico es destacable" (p. 83). Al ser una web gratuita se encuentra al alcance de toda la comunidad educativa, presenta una estructura de manejo intuitivo misma que ayuda para la publicación de diversas aplicaciones en diferentes áreas o fines. El sitio web App Inventor a través de su interfaz, crea archivos con extensión apk (formato de archivos para un concreto sistema o programa) basados en el Sistema Operativo Android. Para complementar, Borrego (2012) menciona que "un Sistema Operativo Android está presente en dispositivos móviles como teléfonos inteligentes, 
relojes, automóviles, tabletas y televisores" (p.2). Al estar presente en los diferentes dispositivos, resulta un punto a favor ya que la gran parte de usuarios cuenta con este tipo de software en su móvil. Es así que Statista (2018) complementa que "Android se consolida como uno de los sistemas operativos más utilizados en el mundo para teléfonos inteligentes con más de 2000 dispositivos en el mundo y más de tres millones de aplicaciones" (p.1). En general este sistema operativo es más accesible en comparación con IOS y Microsoft, debido a la gran cantidad de aplicaciones gratuitas que se pueden encontrar.

Es importante adaptar nuevas metodologías de aprendizaje basadas en las aplicaciones móviles, debido a que se evidencia un nivel de conocimiento básico en respecto al área de Matemática. Por ello EL UNIVERSO (2019) redacta "el 70,9\% de los estudiantes de Ecuador no alcanzó en Matemáticas el nivel 2, categorizado como el nivel de desempeño básico" (p.8). Las estadísticas demuestran un escaso conocimiento de las bases Matemáticas en los estudiantes, aspectos que posteriormente presentarán dificultades de aprendizaje para elementos mucho más complejos. Para complementar las estadísticas de las prueba PISA-D realizadas en 2018, Radio Huancavilca (2019) redacta "En Ecuador, el 49 \% de los estudiantes alcanzó el nivel mínimo de Competencia en Lectura, el 29 \% en Matemáticas y el $43 \%$ en Ciencias" (p.1). Observamos que es preocupante el nivel mostrado por los estudiantes, esto en tres materias que son primordiales para el futuro estudiantil.

La idea de esta investigación nace de la necesidad de aplicar nuevas metodologías de aprendizaje enfocadas en la utilización de aplicaciones móviles. Es así que se hace inclusión de la gamificación como punto de evaluación y refuerzo de los conocimientos. Para profundizar el termino, Gaitán (2013) define "la Gamificación es una técnica de aprendizaje que traslada la mecánica de los juegos al ámbito educativo-profesional con el fin de conseguir mejores resultados (p.1). Al presentar una mecánica dinámica se aumenta el atractivo de la aplicación móvil, así como también se potencializa la finalidad de la misma.

Este artículo se encuentra estructurado de la siguiente manera. En la sección 2 con trabajos previos obtenidos en base la investigación bibliográfica de sitios web. En la sección 3 se presenta conceptos relacionados con el presente estudio. En la sección 4 se muestra la metodología implementada para el correcto desarrollo del trabajo investigativo y los resultados de accesibilidad a dispositivos móviles y aplicaciones por parte de los estudiantes. En la sección 5, se evidencia la propuesta tecnológica. Finalmente, en la sección 6 , se cierra con las conclusiones obtenidas con base en los resultados.

\section{Trabajos relacionados}

Un estudio en el año 2015, en la Universidad de Salamanca "España" propone una innovación docente enfocada en la aplicación de App Inventor para la creación de aplicaciones didácticas para matemáticas. En los resultados obtenidos de 17 estudiantes se verifica que el $64 \%$ siente atracción por la asignatura y un $41 \%$ asegura un cambio en su actitud por las matemáticas. Los autores concluyen que la tecnología App Inventor es accesible y potente para la creación de aplicaciones de contenido didáctico que ayudan a mejorar la actitud y aprendizaje de los alumnos (Almaraz, et al. 2015).

Un proyecto técnico en 2016, propone el diseño e implementación de una aplicación móvil que facilite el proceso de aprendizaje del lenguaje de los niños con Síndrome de Down de la Fundación FASINARM de Guayaquil. Se realizaron pruebas de aceptación por parte de los niños para con la aplicación en donde los resultados fueron totalmente positivos. De acuerdo a los resultados obtenidos los autores concluyen que la aplicación ha sido beneficiosa para los niños y niñas del nivel inicial II, puesto que les ha favorecido en el proceso de aprendizaje (Aranda y Samaniego, 2016). 
En el año 2017, se produce una investigación en la Universidad Politécnica "Venezuela" para observar la factibilidad de implementación de la herramienta App Inventor para el aprendizaje de algoritmos, se realiza el estudio tomando en cuenta a estudiantes de la carrera de Ingeniería Informática. Los resultados correspondientes a la necesidad de aprendizaje evidencian que la forma de manifestación y el alcance de aprendizaje son importantes para el estudiante. Así mismo en la Cuadro de dispositivos más utilizados por el estudiante, el teléfono celular se lleva una ponderación alta. Por tanto concluyen los autores que la herramienta App Inventor es factible para usarla como herramienta de aprendizaje dentro de la unidad curricular (Jiménez y Larrea, 2017).

Una investigación en el año 2018, busca el aprendizaje introductorio de programación de computadoras utilizando App Inventor en un Instituto de Guatemala. Se realizó una evaluación diagnóstica a 18 estudiantes en donde se presentaban 4 situaciones diferentes en donde con un 33\% es el valor más alto alcanzado, después de tomar el curso la valoración obtenida es de 55\% evidenciando una mejoría. A partir de los resultados el autor concluye que el curso introductorio utilizando App inventor ha favorecido el desarrollo de pensamiento crítico y razonamiento en los estudiantes (Valdez, 2018).

En el año 2018, un proyecto de innovación docente en la Universidad de Cádiz "España" desarrolla la App VectorialZ para la visualización interactiva de gráficos creados con un software basado en App Inventor. Se recogieron aspectos de aceptación a través de una encuesta a 24 alumnos, en donde los resultados fueron positivos en aceptación y utilidad, además se evidencia que la mayoría de usuarios ingreso a la App fuera del horario de clase. Los autores concluyen que la evaluación de la App VectorialZ es prometedora ya que los estudiantes en su mayoría destacaron que facilita el aprendizaje de contenidos (Listán, et al. 2018).

En el año 2018, en Colombia, se presenta un objeto virtual de aprendizaje de las operaciones adición y sustracción de números enteros. Las pruebas realizadas contaron con dos grupos, el primero trabajó con el modelo de aprendizaje tradicional presentando resultados deficientes, mientras el segundo grupo usó el objeto virtual de aprendizaje, obteniendo una mejor ponderación. En base a los resultados el autor concluye que el objeto virtual obtuvo mejores resultados académicos tanto en la comprensión de las operaciones, como en su uso para la solución en situaciones similares (García, 2018).

En el año 2018, se presenta en la Universidad Pública de Navarra "España” una propuesta de aprendizaje para estudiantes del siglo XXI, proyecto colaborativo basado en App Inventor. En la encuesta realizada a 42 alumnos, el $40 \%$ ve difícil la programación mediante esta herramienta, además el $95,2 \%$ cree que es importante realizar nuevas versiones de aplicaciones móviles. El autor concluye que el proyecto cumple con las expectativas, ya que el uso del dispositivo móvil ayuda al estudiante a crear un aprendizaje activo fomentando un conocimiento real alejándolo del aprendizaje pasivo (Sanz, 2018).

En el año 2019 se propone una herramienta tecnológica para el aprendizaje lúdico de la matemática, en la Universidad Tecnológica Indoamérica "Ecuador". Mediante la encuesta realizada a los estudiantes se observa que un $65 \%$ muestra bajo rendimiento académico en matemática y un $85 \%$ está abierto al aprendizaje mediante la aplicación móvil presentada. Mediante los resultados el autor finaliza que la aplicación cumple con los objetivos ya que potenció el desarrollo significativo y a su vez fortaleció la adquisición de destrezas con criterio de desempeño (Pérez, 2019). 


\section{Conceptos Relacionados}

\subsection{Aprendizaje}

El aprendizaje se interrelaciona con el término de enseñanza, ya que se desglosa de una manera u otra de ella. Para complementar, Sánchez (2003) define el aprendizaje como "un proceso de naturaleza extremadamente compleja, cuya esencia es la adquisición de un nuevo conocimiento, habilidad o capacidad" (p. 9). Esta adquisición de conocimientos se realiza a través de diferentes medios, con el objetivo principal de que no sea una retención pasajera. Este conocimiento para ser considerado aprendizaje, debe ayudar a futuro a la resolución de problemáticas de la vida cotidiana.

\subsection{Aprendizaje significativo}

Actualmente el aprendizaje significativo es muy importante en el ser humano, para la creación y construcción de nuevos conocimientos. Un aprendizaje es significativo, según Ausubel (1983) cuando:

Los contenidos son relacionados de modo no arbitrario y sustancial (no al pie de la letra) con lo que el alumno ya sabe. Por relación sustancial y no arbitraria se debe entender que las ideas se relacionan con algún aspecto existente específicamente relevante de la estructura cognoscitiva del alumno, como una imagen, un símbolo ya significativo, un concepto o una proposición (p. 2).

Al hacer mención a una estructura cognoscitiva, se deduce a la comprensión y memoria que conforman la estructura del conocimiento de una persona. Es decir, las ideas conjuntamente relacionadas, en referencia a un conocimiento y saber de determinado tema o concepto.

\subsection{Aplicación Móvil}

Se conoce como aplicación móvil (App) a todo aquel programa que se encuentra en un dispositivo móvil o tableta. Al respecto, Enríquez y Casas (2013) señalan que:
Aplicación móvil es aquel software desarrollado para dispositivos móviles. Móvil se refiere a poder acceder desde cualquier lugar y momento a los datos, las aplicaciones y los dispositivos. Este tipo de aplicaciones se desarrollan teniendo en cuenta las limitaciones de los propios dispositivos, como por ejemplo el bajo poder de cómputo, la escasa capacidad de almacenamiento, ancho de banda limitado, entre otros (p.35).

Cada una de las aplicaciones móviles instaladas en un dispositivo celular posee una serie de requerimientos, esto dependiendo la finalidad que tenga el programa. Los requisitos se dan con base en el tamaño de almacenamiento que varía por la cantidad de contenido gráfico como: imágenes, sonidos, videos entre otros.

Según Artica (2014), menciona que: "las aplicaciones nacen de alguna necesidad concreta de los usuarios, y se usan para facilitar o permitir la ejecución de ciertas tareas en las que un analista o un programador han detectado una cierta necesidad" (p.3). Cada una de las necesidades sirve para facilitar el cumplimiento de una actividad y en base a su finalidad Medina (2018) las engloba de la siguiente manera como se muestra en la Figura 1: 


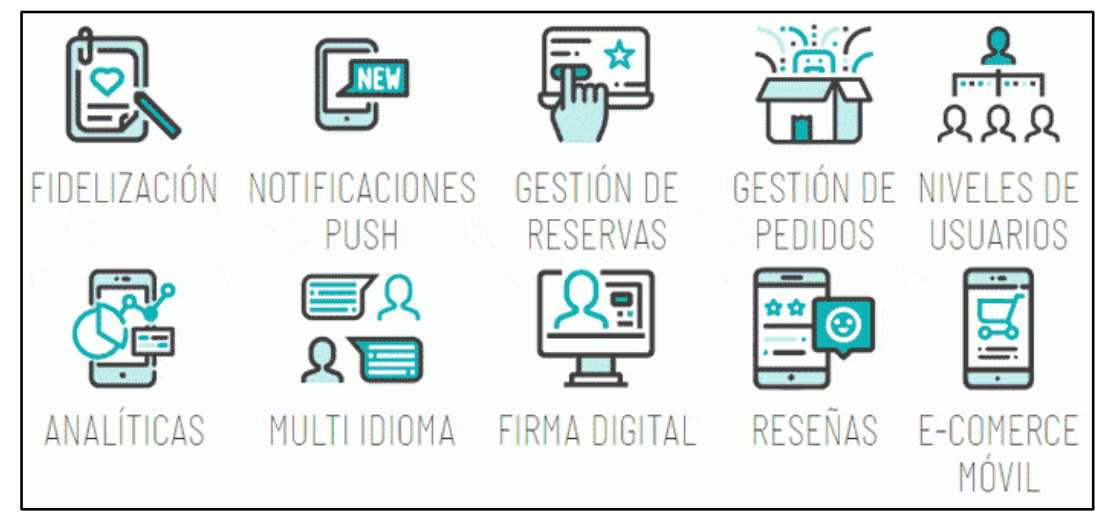

Figura 1. Tipos de aplicaciones móviles (Medina, 2018, p. 1).

Todas las aplicaciones móviles cumplen con características y funciones específicas según su propósito de creación. Cada una de ellas satisfaciendo la necesidad que presente el usuario en variados campos. Entre el grupo de aplicaciones se refleja el uso de aplicaciones móviles para finalidades como: el entretenimiento, trabajo, ocio, comunicación, salud, etc.

\subsection{App Inventor}

Es un espacio web libre que motiva a la creación de contenido. La página oficial de MIT App Inventor (2012) la define como "un entorno de programación visual e intuitivo que permite a todos, incluso a los niños, crear aplicaciones totalmente funcionales para teléfonos inteligentes y tabletas" (p.1). Con lo antes mencionado se conoce que App Inventor ${ }^{1}$ es de simple manejo y ayuda al diseño de aplicaciones Android para diferentes propósitos de la vida cotidiana.

Estos propósitos pueden ser para trabajo, ocio, noticias, educación entre otros según la finalidad que busque el creador. Al respecto, Posada (2008) menciona que "ofrece a sus usuarios/as una interesante solución tecnológica con la cual crear App para dispositivos Android de una forma sencilla" (p.3). Este medio web al ser una nueva puerta digital para niños, jóvenes y adultos quiere erradicar el consumo de tecnología y busca promover la creación de la misma.

La creación e innovación de tecnología ayuda al usuario, ya que beneficia al pensamiento lógico del creador al momento del diseño de cada una de las aplicaciones. Estas Apps al ser compatibles con Android pueden instalarse fácilmente en un cualquier dispositivo móvil que cuente con este sistema operativo.

\subsection{Gamificación}

Dentro del ámbito educativo se puede evidenciar la aplicación de nuevas metodologías para mejorar el aprendizaje del alumno una de ellas es la gamificación. Término que Gaitán (2013) define como:

Una técnica de aprendizaje que traslada la mecánica de los juegos al ámbito educativo-profesional con el fin de conseguir mejores resultados, ya sea para absorber mejor algunos conocimientos, mejorar alguna habilidad, o bien recompensar acciones concretas, entre otros muchos objetivos (p.1). 
Ante lo mencionado se pueden usar varios elementos que sirvan como recurso educativo, para realizar juegos que ayuden a estimular al estudiante a la resolución de problemas y construcción de pensamiento crítico mediante una problemática de la vida cotidiana.

\subsection{Números enteros}

Los números enteros nacen, por la idea de facilitar los cálculos respecto a números que estén por debajo del cero y obtener resultados más precisos a la realidad. Según Rumiche (2019), define que "la necesidad de representar cantidades menores que cero, y hacer siempre posible la sustracción motivó la creación del conjunto de los números enteros (Z)" (p.17). Es así que los números enteros Z, están conformados por los siguientes componentes y símbolo: números enteros positivos $\left(Z^{+}\right)$, números enteros negativos $\left(Z^{-}\right)$y el cero. Una vez teniendo clara las definiciones de aprendizaje, a continuación se presenta una lista ordenada para el correcto ciclo de aprendizaje de números enteros.

1. Preparación de temas base para el estudio de números enteros.

2. Brindar recursos y materiales que contengan teoría y propiedades con números enteros.

3. El estudiante revisa y capta cada una de las operaciones presentadas.

4. Asimilación de la información y puesta en práctica en ejercicios y problemas de aplicación.

En cada una de las etapas cada actor educativo cumple un papel importante, en primera instancia el maestro que es quien guía para que se cumplan con lo planteado. Y por otro lado el estudiante que es quien recibe cada uno de los conocimientos, para posteriormente ponerlos en práctica. Esto mediante un elemento medio que son los recursos educativos, que sirven de puente para la relación entre maestro-estudiante.

\section{Metodología}

El trabajo de investigación se realizó mediante la metodología que se detalla a continuación:

- Enfoque: El trabajo de investigación se realizó mediante el enfoque mixto (cualitativo y cuantitativo), debido a la identificación de la evidente problemática que presenta el aprendizaje de Matemática, específicamente en la resolución y comprensión de operaciones con números enteros. Toda esta información fundamentada por datos medibles recolectados en la institución educativa; a través, de un instrumento previamente validado por expertos en investigación. Para complementar según Hernández (2014) "El enfoque mixto constituye una posible elección para enfrentar problemas de investigación y resulta igualmente valioso. Es, hasta ahora, la mejor forma diseñada por la humanidad para investigar y generar conocimientos" (p.2).

- Tipo de investigación: El proyecto utilizará una investigación de campo; a través, de la recolección de datos provenientes de las encuestas elaboradas en el área de Matemática. Este instrumento está dirigida a los estudiantes de Octavo Grado de Educación General Básica, en la escuela de Educación Básica Eugenio de Santa Cruz y Espejo.

También se manejará la investigación documental para la elaboración del marco teórico, con base en la recopilación de información. Todos los datos serán seleccionados mediante su búsqueda en diferentes fuentes documentales, las cuales servirán para complementar la información del objeto de estudio.

- Nivel: Hernández (2014) menciona "los estudios exploratorios se realizan cuando el objetivo es examinar un tema o problema de investigación poco estudiado, del 
cual se tienen muchas dudas o no se ha abordado antes" (p.91). Es así que para la elaboración del trabajo investigativo se optó por el nivel exploratorio, en cuanto a que en la institución educativa eran poco conocidas y exploradas las aplicaciones móviles. En específico su uso como recurso didáctico para el aprendizaje de operaciones matemáticas con números enteros mediante actividades interactivas e innovadoras.

A la par se utilizó el nivel descriptivo, permitiendo apreciar el estado real del octavo grado en su formación de Matemática, especialmente en la comprensión y resolución de operaciones con números enteros. Con esta apreciación se ayudó a examinar el entorno educativo, los recursos didácticos que disponen en la institución y algunas de las dificultades de aprendizaje. Y así encontrar una solución a través del diseño de una aplicación móvil educativa a través de App Inventor; que sirva como herramienta pedagógica innovadora para el estudiante. Con base en lo mencionado Hernández (2014) acota que "con los estudios descriptivos se busca especificar las propiedades, las características y los perfiles de personas, grupos, comunidades, procesos, objetos o cualquier otro fenómeno que se someta a un análisis" (p.92).

- Técnica: Como técnica para la obtención de información y datos para la presente investigación se ha optado por la encuesta. Esta encuesta será realizada de manera online, con la ayuda de la plataforma en línea Google Forms.

- Instrumento: El cuestionario se realizó tomando en cuenta factores de composición y coherencia dentro de la aplicación móvil. Con base en lo expuesto el cuestionario presentado contiene 15 preguntas, en donde su elección está compuesta por 2 respuestas, una positiva y una negativa. En donde se puede observar las opciones de SÍ APLICA o en su contra NO APLICA, según la perspectiva del usuario.

- Validez: Para la validez del instrumento de recolección de datos, se contó con la ayuda de dos docentes de la Facultad de Filosofía, Letras y Ciencias de la Educación de la carrera de Pedagogía de las Ciencias Experimentales Informática. Se analizó a través de una matriz calificar la correspondencia, calidad y lenguaje de cada uno de los ítems.

\subsection{Población y muestra}

En el presente proyecto de investigación, la población seleccionada está compuesta por las y los estudiantes de Octavo año de Educación General Básica, pertenecientes a la Escuela de Educación Básica Eugenio de Santa Cruz y Espejo. En el Cuadro 1 se aprecia de manera exacta cuantos estudiantes fueron seleccionados para encuestar.

\begin{tabular}{ll}
\hline Población & Cantidad \\
\hline Octavo EGB "A" & 33 \\
Octavo EGB "B" & 31 \\
Octavo EGB "C" & 30 \\
Total & 94 \\
\hline
\end{tabular}

Cuadro 1. Población Octavo EGB

\subsection{Resultados}

Los resultados que se presentan en los gráficos estadísticos de la Figura 3, corresponden a los primeros cinco ítems del cuestionario aplicado. Las preguntas van enfocadas hacia la facilidad de la utilización de la aplicación móvil educativa, desde el punto de vista del usuario. 


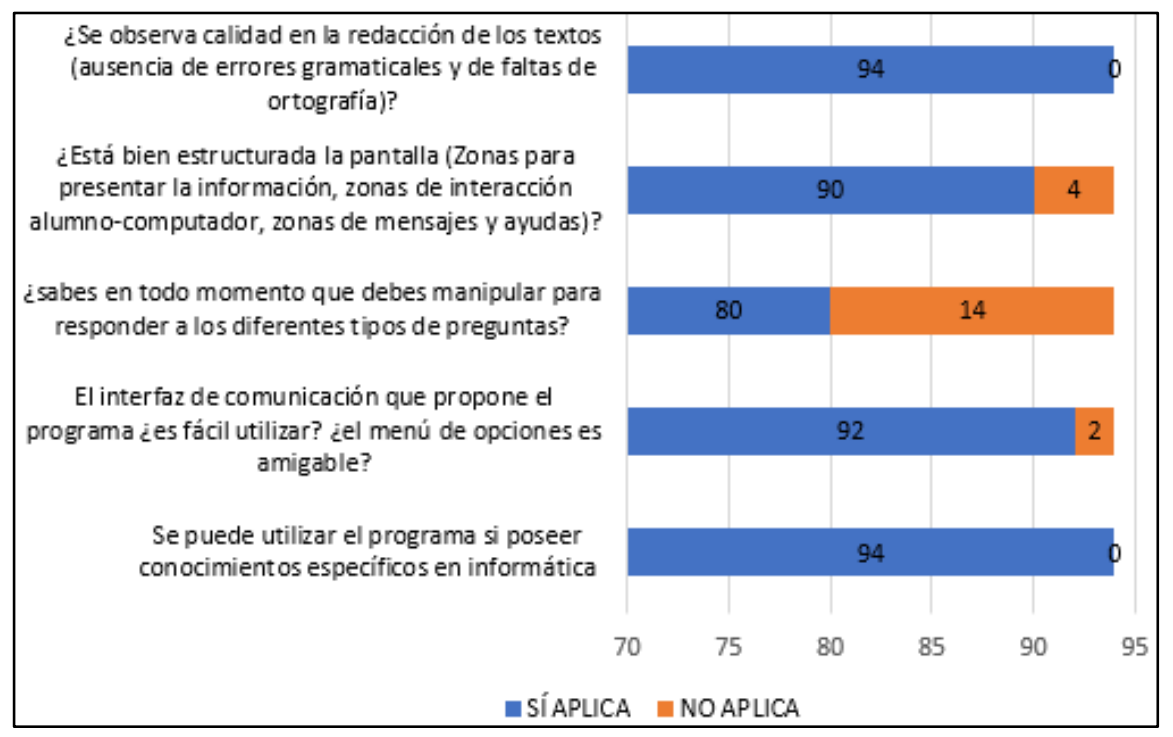

Figura 2. Resultados de utilización del programa

Con base en los resultados de la primera sección, se visualizan aspectos positivos en cuanto a la facilidad de manipulación de la herramienta por parte del estudiante. Así mismo se establece que el diseño y sus componentes están en su mayoría muy bien estructurados, ya que permiten que su manejo sea intuitivo e interesante para el alumno.

A continuación en la Figura 4 se aprecia el siguiente bloque de preguntas, relacionados a aspectos de la visualización dentro de la aplicación móvil educativa. Esto tiene que ver con elementos, formas, tamaños, colores, entre otros que conforman el entorno virtual del programa.

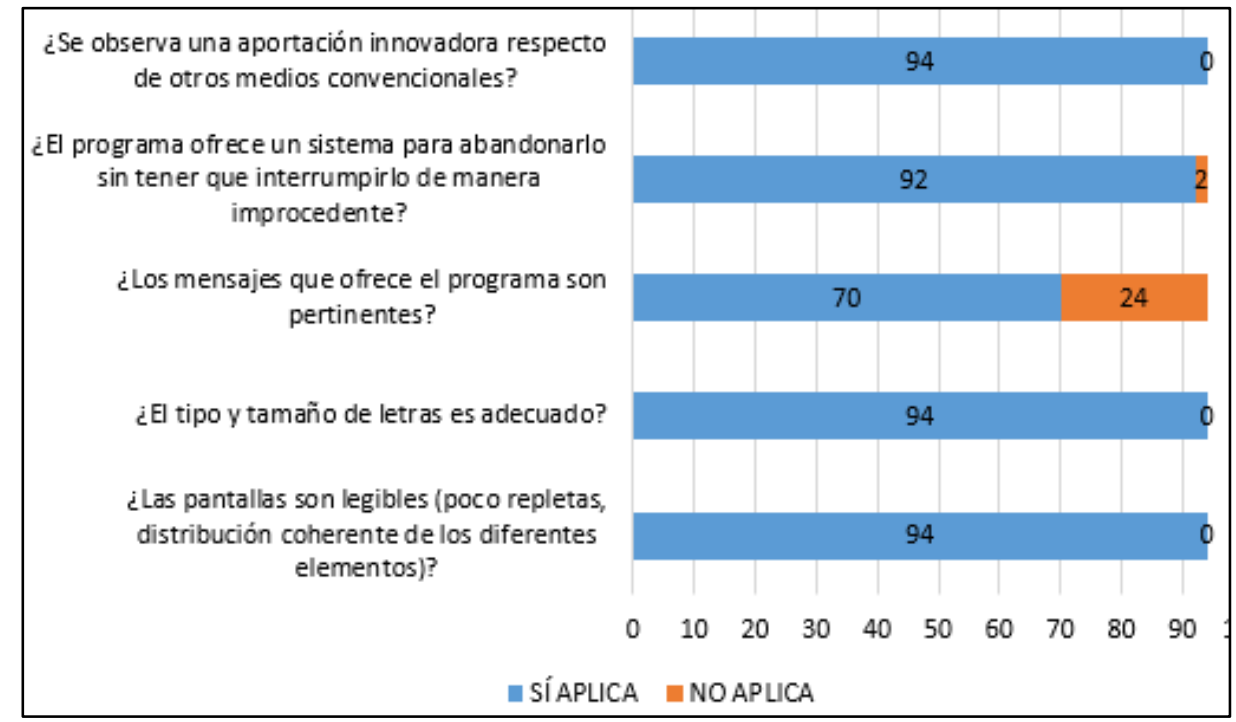

Figura 3. Resultados de visualización por pantalla

Los resultados evidencian mayormente respuestas positivas, en cuanto a la conformación y estructuración del contenido y su forma dentro de la App Móvil. Solamente una escasa parte de la población no considera la pertinencia de algunos elementos, así como también insinúan la inclusión de otros botones de acción para mayor facilidad de navegación entre pantallas. 
En el último apartado del cuestionario Figura 5, se encontró ítems que fueron dirigidos a la interacción que existe entre el usuario y la aplicación como tal. Esto con el fin de demostrar que el aprendizaje no será pasajero, sino dinámico e innovador.

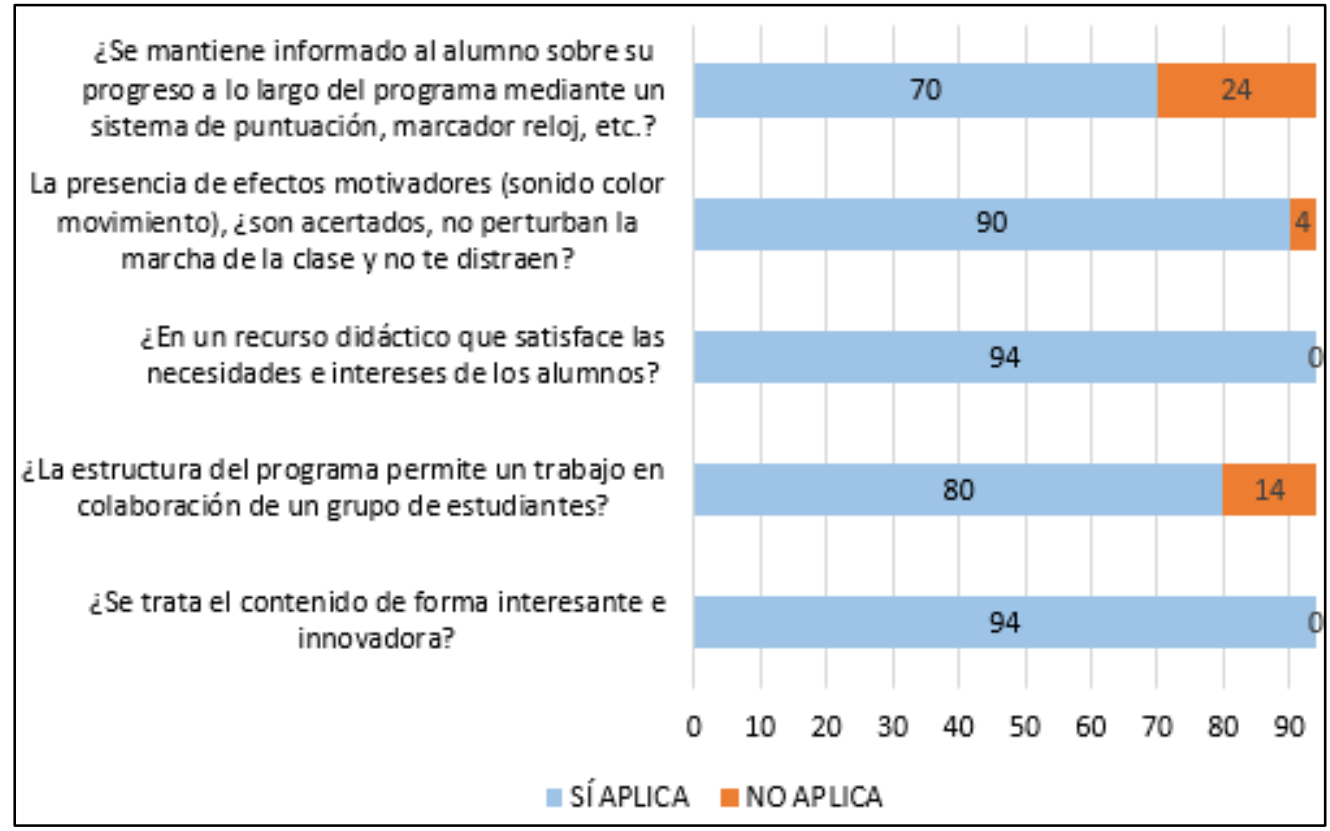

Figura 4. Resultados de formas de interacción

Con base en resultados obtenidos en las principales preguntas de la encuesta, se puede evidenciar que en su mayoría las respuestas de los estudiantes favorecen al proyecto tecnológico. Es así que tomando todas las apreciaciones de los usuarios se ha construido una App móvil educativa que cubra las necesidades del nivel de conocimiento al cual pertenecen. Aportando con un juego de memoria, que permita al estudiante poner en práctica los conocimientos estudiados en la aplicación móvil educativa.

\section{Implementación}

Para la correcta instalación y funcionamiento de la aplicación MatEstudio, el dispositivo móvil debe contar con los mínimos requisitos que se detallan en el Cuadro 3. Esto para evitar fallos en el transcurso de operación del programa, así como también fallas de visualización en el diseño final en algunos equipos que no cuenten con estas características.

\begin{tabular}{ll}
\hline Sistema Operativo & Android Versión 7 o superior \\
\hline Almacenamiento & 12,5 MB de memoria libre \\
\hline RAM & 1,6 Gb o superior \\
\hline Pantalla & $640 \times 1136$ \\
\hline
\end{tabular}

Cuadro 2. Componentes Requeridos del Programa

Para acceder a la aplicación, se procedió a subir al sistema de almacenamiento Google Drive como se visualiza en la Figura 6 el archivo MatEstudio.apk. Mediante a internet se dirige al link $^{2}$ correspondiente de almacenamiento del archivo. 


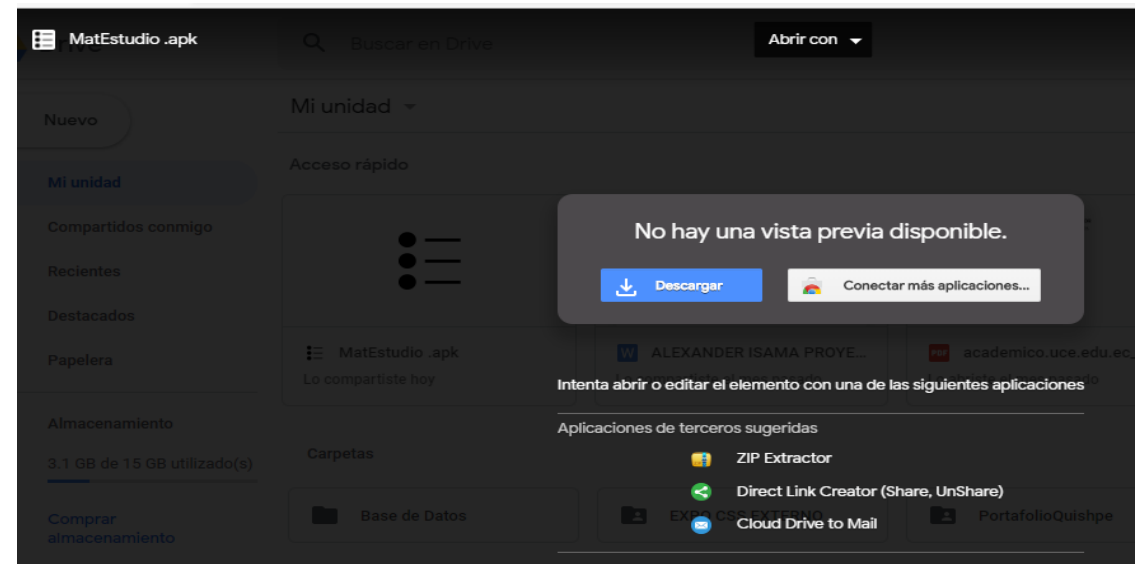

Figura 5. Sitio de descarga del programa

La aplicación como tal presenta diferentes pantallas, todas llamativas y de colores dinámicos para llamar la atención del usuario. En la Figura 7 se visualiza lo antes mencionado, tenemos las pantallas de Bienvenida y el menú donde el usuario decide en cuál de las operaciones desea ingresar para ver su contenido. Como se visualiza se presentan 8 opciones de pantalla, las cuales contienen cada una de las operaciones matemáticas esenciales, así como también una introducción general al tema y por último el juego antes mencionado.

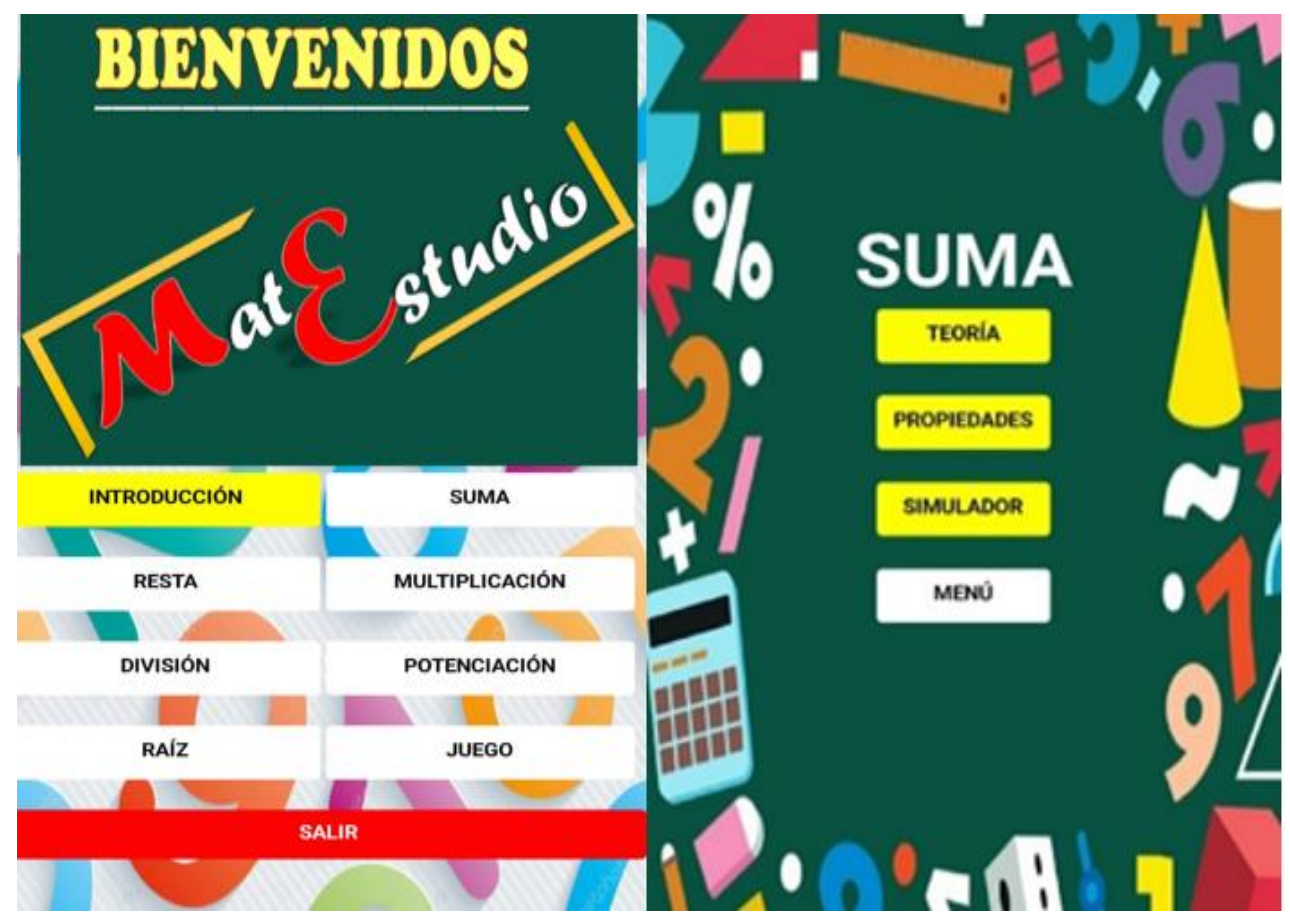

Figura 6. Pantalla de Bienvenida y Menús

A continuación, al estar ya dentro de cualquier operación se presentan submenús que presentan teoría, propiedades y simuladores de la operación señalada. En la Figura 8 se

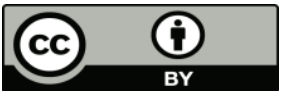


puede observar el contenido y a su vez los simuladores de operación en donde se puede poner en práctica los conocimientos ya antes estudiados.

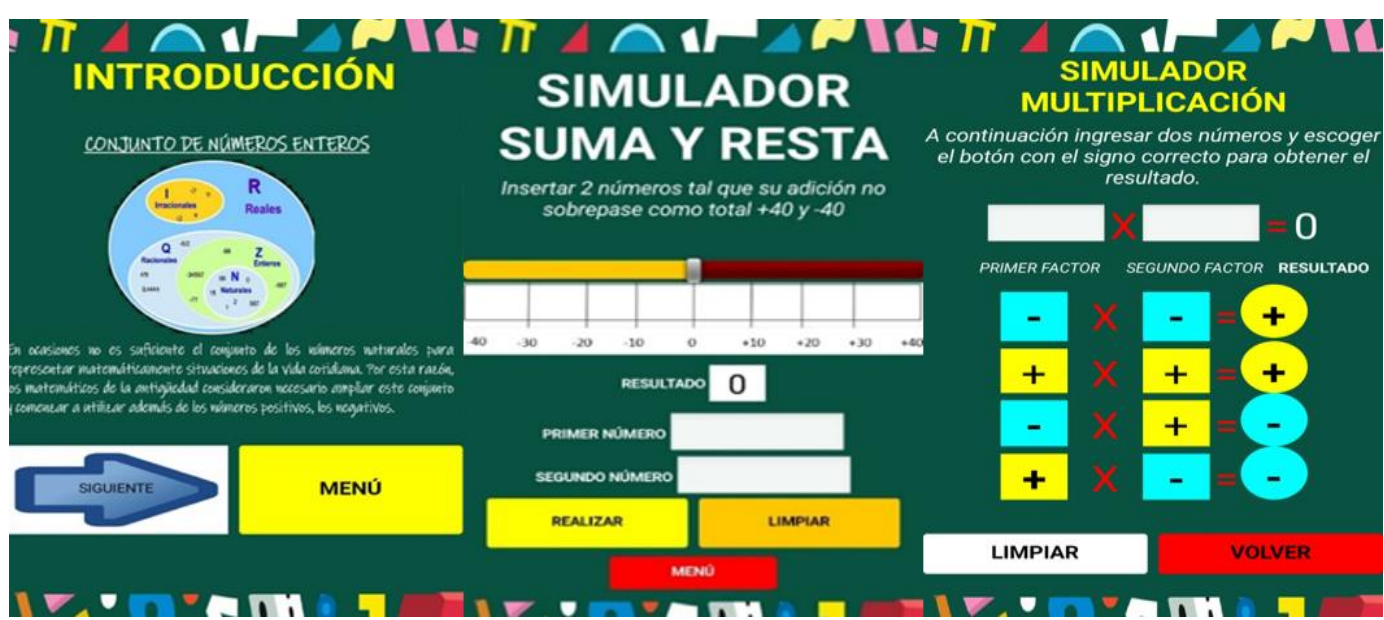

Figura 7. Pantalla de Teoría y Simuladores

Finalmente, para poner en práctica los conocimientos vistos en cada una de las pantallas, se ha incluido un juego de memoria. En la Figura 9 se visualiza que está compuesto por 16 botones de acciones en los cuales el alumno tiene un tiempo límite para poder resolverlo.

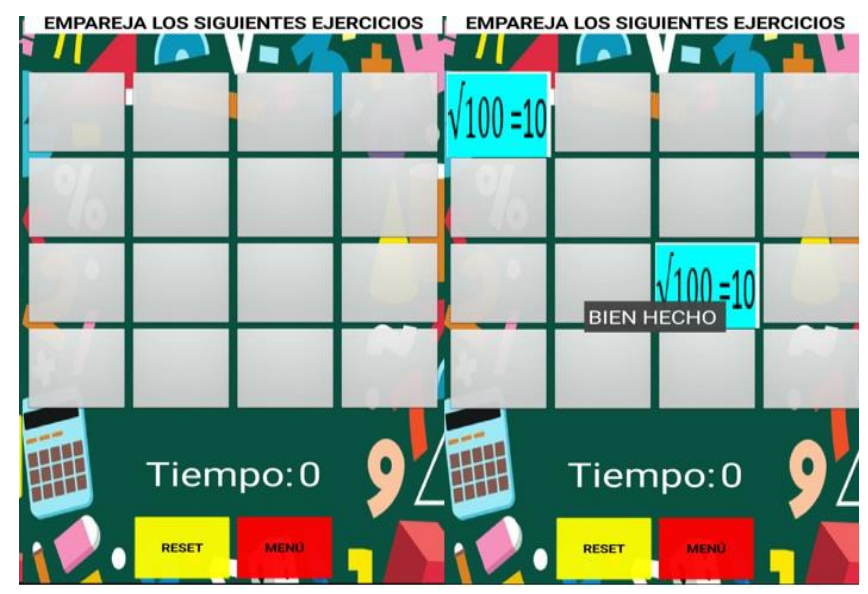

Figura 8. Pantalla de Juego

El nuevo sistema cuenta con una pantalla de juego de memoria con el fin de estimular y motivar de mejor manera el uso de la aplicación móvil para reforzar el aprendizaje de números enteros. Apartado que contiene parte de la teoría de las diferentes operaciones matemáticas, pero que son puestas en práctica al momento de la resolución de un ejercicio propuesto.

\section{Conclusiones}

Con la presente investigación se ha podido diseñar de manera innovadora una nueva herramienta educativa tecnológica, conformada por elementos dinámicos dentro de un entorno educativo que ayudan al aprendizaje. Además, se pudo verificar la factibilidad tanto económica como tecnológica para el estudio de operaciones con números enteros a través de una aplicación móvil educativa. Para lo cual, se diseñaron varias pantallas con contenido y colores llamativos, así como también la inclusión de un juego dentro del mismo programa 
con la finalidad de satisfacer todas las necesidades del estudiante, acorde al nivel de educación al cual pertenece.

El uso de aplicaciones móviles en la actualidad se encuentra en plena tendencia, la posibilidad de aprovechar cada una de ellas depende del buen manejo de la misma realizado por cada usuario. Esto siguiendo tutoriales y manuales de uso, para aprovechar al máximo cada una de las operaciones que nos permiten realizar este tipo de programas móviles.

Las aplicaciones móviles como todo programa creado pueden traer variantes y mejoras con el pasar del tiempo. Con base a seguimientos realizados y evaluando resultados de uso, se puede presentar cambios ya sea en la estructura, así como también en el contenido de la aplicación, con la finalidad de brindar un producto de calidad que pueda expandirse a más grupos de usuarios. 


\section{Bibliografía}

Almaraz, F., Maz, A., y López, C. (2016). Tecnología móvil y enseñanza de las matemáticas. Revista EPSILON. https://thales.cica.es/epsilon/?q=content/tecnología-móvil-yenseñanza-de-las-matemáticas-una-experiencia-de-aplicación-de-app-invento

Almaraz, J; Campos, P y Castelo, T. (2015). Desarrollo de una aplicación Web para la gestión de Entornos Virtuales. https://eprints.ucm.es/13083/1/Memoria SI Final.pdf

Aranda, C., y Samaniego, V. (Abril de 2016). Análisis, diseño e implementación de una aplicación móvil que facilite el proceso de enseñanza-aprendizaje del lenguaje de los niños con síndrome de Down del inicial II de la fundación FASINARM de Guayaquil. Repositorio UPS. https://dspace.ups.edu.ec/bitstream/123456789/12636/1/UPSGT001658.pdf

Artica, R. (2014). Desarrollo de Aplicaciones Móviles. http://repositorio.unapiquitos.edu.pe/bitstream/handle/UNAP/4515/Robertho Te sis Titulo 2014.pdf? sequence $=1$ \&isAllowed $=\mathrm{y}$

Ausubel, D. (1983). Teoría del aprendizaje significativo. Fascículos de CEIF, 1(1-10). http://www.academia.edu/download/38902537/Aprendizaje significativo.pdf

Borrego, Á. (2012). Introducción a Android. 1-22

EL UNIVERSO. (2019). Ecuador reprobó en matemáticas en evaluación internacional. https://www.eluniverso.com/guayaquil/2019/02/26/nota/7207946/matematicas -no-se-paso-prueba

Enríquez, J y Casas, S. (2013). Usabilidad en aplicaciones móviles. https://dialnet.unirioja.es/descarga/articulo/5123524.pdf

Gaitán, V. (2013). Gamificación: el aprendizaje divertido.

García, D. (2018). Objeto virtual de aprendizaje de las operaciones adición y sustracción de números enteros. universidad pedagógica nacional. http://hdl.handle.net/20.500.12209/10235

Hernández, R. (2014). Metodología de la Investigación. Cuarta Edición. México. Interamericana Editores, S.A.

http://200.11.208.195/blogRedDocente/alexisduran/wpcontent/uploads/2015/11/CON FIABILIDAD.pdf

Jiménez, N., y Larrea, A. (2017). Aplicación informática (App Inventor): Herramienta para el aprendizaje de algoritmos. Revista RECITIUTM. http://www.recitiutm.iutm.edu.ve/index.php/recitiutm

Listán, M., Mota, J., Muriel, M., Barreno, F., Ruiz, A., y Vidal, J. (2018). Desarrollo de la app VectorialZ para la visualización interactiva de gráficos creados con software matemático. https://indoc.uca.es/articulos/sol-201700083572-tra.pdf

Medina, J. (2018). Aplicaciones Móviles ¿Vale la pena para tu empresa? Emprendedor en la nube. $\quad$ https://www.emprendedorenlanube.com/moviles/son-necesarias-lasaplicaciones-moviles.php 
MIT. (2012). MIT APP INVENTOR. http://appinventor.mit.edu/about-us

Pérez, J. (2019). HERRAMIENTAS TECNOLÓGICAS PARA EL APRENDIZAJE LÚDICO DE LA MATEMÁTICA EN LOS ESTUDIANTES DE NOVENO DE EDUCACIÓN GENERAL BÁSICA SUPERIOR DEL COLEGIO DE BACHILLERATO "CHAMBO". http://repositorio.uti.edu.ec//handle/123456789/1353

Posada, F. (2008). Creando aplicaciones para móviles Android con App Inventor 2. https://intef.es/wp-content/uploads/2019/03/MIT-App-Inventor-2.pdf

Radio Huancavilca. (2019). Ecuador reprobó matemáticas en evaluación internacional. https://radiohuancavilca.com.ec/ecuador-reprobo-en-matematicas-en-evaluacioninternacional/

Rumiche, C. (2019). Ecuaciones e inecuaciones en el conjunto de los números enteros. http://dspace.unitru.edu.pe/handle/UNITRU/14838

Sánchez, I. (2003). Elementos conceptuales básicos del proceso de enseñanzaaprendizaje. ACIMED, Ciudad de La Habana, v. 11, n. 6, dic. 2003. http://scielo.sld.cu/scielo.php?script=sci arttext\&pid=S102494352003000600018\&lng=es\&nrm=iso

Sanz, I. (2018). Propuesta de aprendizaje para estudiantes del siglo XXI, proyecto colaborativo basado en APP Inventor. https://hdl.handle.net/2454/31154

Statista. (2018). • Mobile OS market share 2018 | Statista. Retrieved May 19, 2019, from https://www.statista.com/statistics/266136/global-market-share-held-bysmartphone-operating-systems/

Valdez, R. (2018). Curso de introducción a la programación de computadoras utilizando el software app inventor en el instituto de cooperación social http://www.repositorio.usac.edu.gt/id/eprint/8741 


\section{Autores}

CRISTHIAN QUISHPE-LÓPEZ Obtuvo el título de Licenciado en Ciencias de la Educación Mención Informática por la Facultad de Filosofía, Letras y Ciencias de la Educación de la Universidad Central del Ecuador en 2020.

SANTIAGO VINUEZA-VINUEZA obtuvo su título de Magíster en Redes de Comunicaciones, de la Facultad de Ingeniería, de la Pontificia Universidad Católica del Ecuador en 2016, Magíster en Sistemas Informáticos Educativos, Universidad Tecnológica Israel en 2009, Licenciado en Ciencias de la Educación especialización de Informática, Facultad de Filosofía Letras y Ciencias de la Educación, Universidad Central del Ecuador en 2002, Ingeniero en Ejecución Informática, Universidad Autónoma de Quito en 2002.

Actualmente es profesor Agregado de la Facultad de Ciencias Económicas de la Universidad del Ecuador. Vocal Principal del Fondo de Cesantía de la Universidad Central del Ecuador, Vocal del H.C. Directivo de la Facultad de Ciencias Económicas de la Universidad Central del Ecuador, Coordinador de la Unidad de Titulación de la Carrera de Finanzas de la Universidad Central del Ecuador, Coordinador de la Asignatura de Sistemas de Información de la Facultad de Ciencias Económicas de la Universidad Central del Ecuador, Sus principales investigaciones se enmarcan en el campo educativo y las Tecnologías de la Información y Comunicación. 